\title{
Ellagitannins and Lignan Glycosides from Balanophora japonica (Balanophoraceae)
}

\author{
Zhi-Hong Jiang, ${ }^{*, a, b}$ Takashi Tanaka, ${ }^{a}$ Hiromi Iwata,,${ }^{a}$ Saori SaKamoto,${ }^{a}$ Yoko Hirose,,${ }^{a}$ and \\ Isao KOUNO $^{a}$ \\ ${ }^{a}$ Department of Molecular Medicinal Sciences, Graduate School of Biomedical Sciences, Nagasaki University; Bunkyo \\ Machi 1-14, Nagasaki 852-8521, Japan: and ${ }^{b}$ School of Chinese Medicine, Hong Kong Baptist University; Kowloon \\ Tong, Hong Kong. $\quad$ Received October 27, 2004; accepted November 24, 2004
}

Three new ellagitannins named balanophotannins $A-C$ having a $1,1^{\prime}-\left(3,3^{\prime}, 4,4^{\prime}\right.$-tetrahydroxy)dibenzofurandicarboxyl group in their molecules and four known lignan glycosides were isolated from the extracts of fresh aboveground and underground parts of a medicinal parasitic plant Balanophora japonica (Balanophoraceae). Their structures were elucidated on the basis of spectral and chemical evidence. Chemotaxonomic significance of the known lignan glycosides in Balanophora japonica was discussed.

Key words Balanophora japonica; Balanophoraceae; balanophotannin; ellagitannin; lignan glycoside

In the previous paper, ${ }^{1)}$ we reported the isolation and structural elucidation of eighteen new and sixteen known acyl glucoses having caffeoyl, coumaroyl and hexahydroxydiphenoyl groups from Balanophora japonica M. (Balanophoraceae) collected in Nagasaki Perfecture, Japan which was used as an antipyretic, an antitode and a hemostatic medicine in China. In a continuation of our studies on the chemical constituents of medicinal parasitic plants, ${ }^{1,2}$ we further isolated three ellagitannins named balanophotannins $\mathrm{A}-\mathrm{C}$ $(\mathbf{1}-\mathbf{3})$ and four known lignan glycosides $(4-7)$ from the fresh aboveground part and underground part of Balanophora japonica $\mathrm{M}$. (Balanophoraceae), respectively. This paper deals with the structural elucidation of these compounds by means of NMR, MS techniques and chemical methods. The chemotaxonomic significance of the lignan glycosides in Balanophora japonica was also briefly discussed.

\section{Results and Disscussion}

Balanophotannins $\mathrm{A}-\mathrm{C}(\mathbf{1}-\mathbf{3})$ were isolated from the EtOAc layer of the methanolic extracts of the fresh aboveground part of Balanophora japonica by a combination of column chromatographies over Toyopearl HW-40, Chromatorex ODS, Sephadex LH-20 and MCI-gel CHP20P. Whereas, four known lignan glycosides were obtained from the methanolic extracts of the fresh underground part by means of silica gel, MCI-gel CHP20P, Sephadex LH-20 and TSK gel Toyopearl HW-40F chromatographies. The chemical structures of four known lignan glycosides, i.e., balanophonin 4- $O$ - $\beta$-D-glucopyranoside $(4),{ }^{3,4)}(-)$-isolariciresinol 4- $O$ - $\beta$-D-glucopyranoside $(5){ }^{2)}{ }^{2}(-)$-pinoresinol $O$ - $\beta$-D-glucopyranoside $(\mathbf{6})^{5,6)}$ and $(-)$-pinoresinol di- $O$ - $\beta$-D-glucopyranoside $\left.(7)^{7}\right)$ were determined by comparing their chemical and physical data with those in the references.

Balanophotannin A (1) was isolated as a tan powder showing positive results to $\mathrm{FeCl}_{3} / \mathrm{EtOH}$ reagent. Its molecular formula was determined to be $\mathrm{C}_{34} \mathrm{H}_{24} \mathrm{O}_{21}$ from the quasimolecular ion peak at $m / z$ : 767 observed in the negative FAB-MS and the result of the elemental analysis. The ${ }^{1} \mathrm{H}$ - and ${ }^{13} \mathrm{C}$ NMR spectra of 1 showed great similarities to those of 1,3di- $O$-galloyl-4,6-( $S$ )-hexahydroxydiphenoyl (HHDP)- $\beta$-Dglucopyranose which was isolated from the aboveground part of Balanophora japonica. ${ }^{1)}$ Taking its molecular formula into account, 1 was deduced to be a dehydrated product of 1,3-di$O$-galloyl-4,6-(S)-HHDP- $\beta$-D-glucopyranose. The ${ }^{1} \mathrm{H}-\mathrm{NMR}$ spectral data of 1 supported the existence of two galloyl groups $(\delta 7.25,7.21)$, a tetra-acylated glucopyranose with a ${ }^{4} \mathrm{C}_{1}$ conformation and an aromatic acyl group. From the chemical shift $(\delta 6.02)$ and the coupling constant $(J=8 \mathrm{~Hz})$ of $\mathrm{H}-1$ of the glucose moiety, it was concluded that the hy-
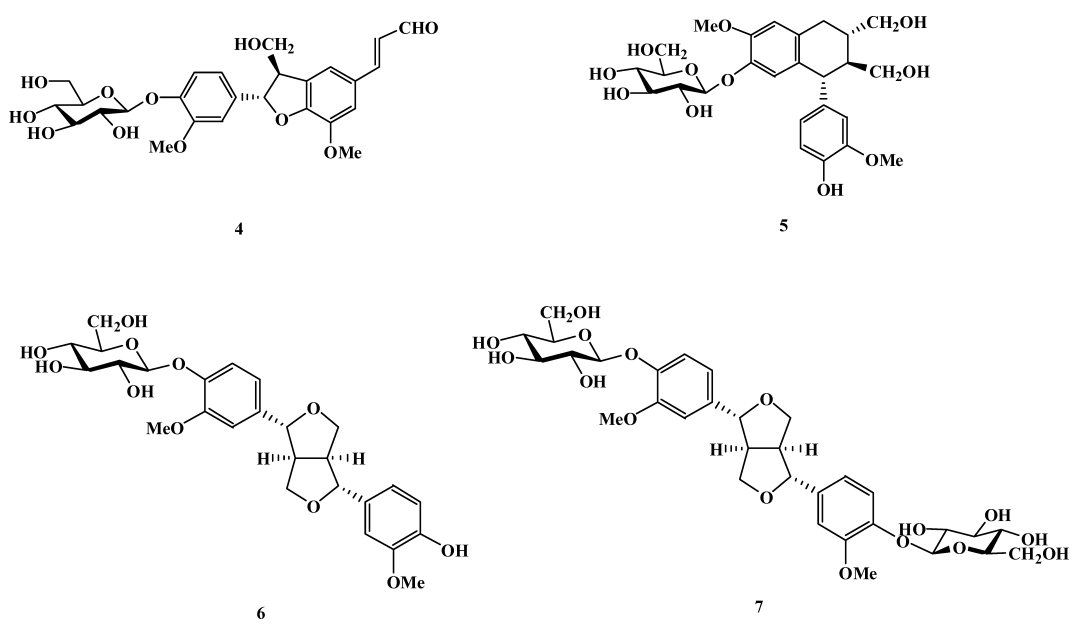

Chart 1 

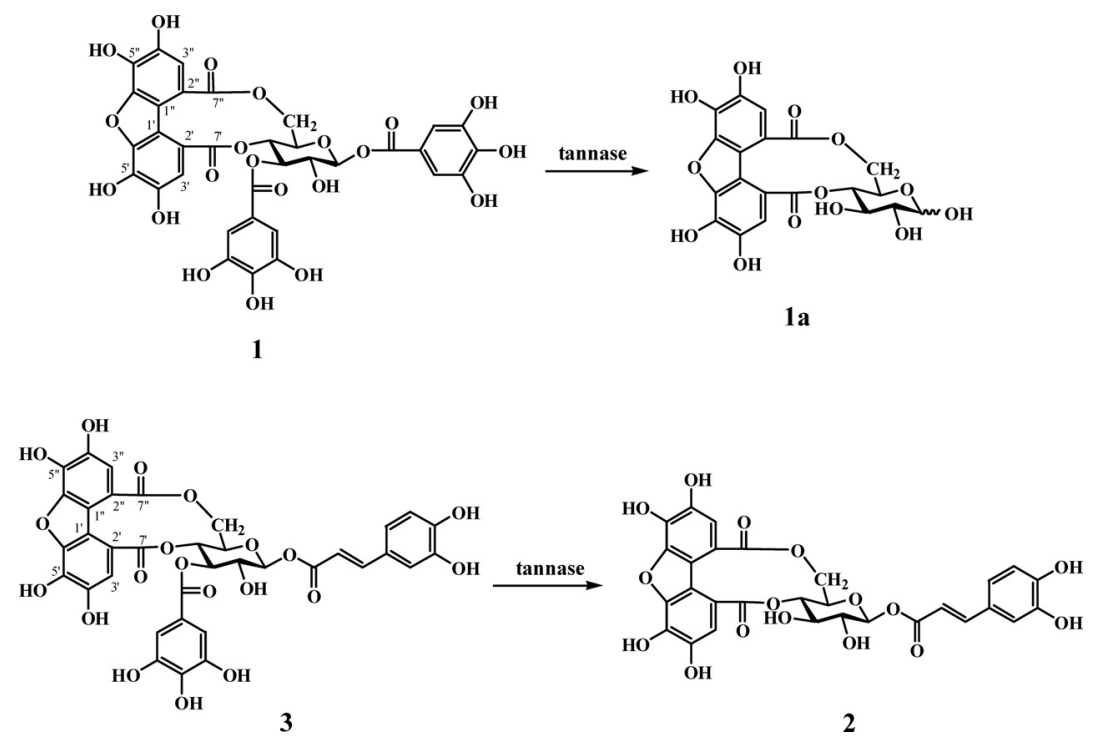

Chart 2

droxyl group of the glucose is acylated and the configuration of the anomeric center is $\beta$ form. A serial of ${ }^{1} \mathrm{H}-{ }^{1} \mathrm{H}$ decoupling experiments led to the assignments of the other proton signals of the glucose moiety, thus confirming that the positions of the other acyl groups are located in C-3, C-4 and C-6 hydroxyls of the glucose moiety. Furthermore, tannase hydrolysis of $\mathbf{1}$ afforded 1a [negative FAB-MS m/z: 463 $\left.[\mathrm{M}-\mathrm{H}]^{-}, \mathrm{C}_{20} \mathrm{H}_{16} \mathrm{O}_{13}\right]$ which was identified as 4,6-acylated glucopyranose from its ${ }^{1} \mathrm{H}-\mathrm{NMR}$ spectral data. Therefore, it was established that two galloyl groups in $\mathbf{1}$ are positioned at $\mathrm{C}-1$ and C-3 hydroxyls of the glucose moiety. In the ${ }^{13} \mathrm{C}-$ NMR spectrum of $\mathbf{1}$ (Table 1), besides the signals assigned to the glucose moiety and galloyl groups, 12 acromatic and two carbonyl signals ${ }^{8,9)}$ were observed, indicating the presence of a $1,1^{\prime}-\left(3,3^{\prime}, 4,4^{\prime}\right.$-tetrahydroxy)dibenzofurandicarboxyl group, which was firstly found to be an acyl group of mallotusinin in Mallotus (Euphorbiaceae) ${ }^{8}{ }^{8}$ On the basis of the above evidence, the structure of balanophotannin A (1) was established to be 1,3-O-di-galloyl-4,6-[1', $1^{\prime \prime}-\left(3^{\prime}, 3^{\prime \prime}, 4^{\prime}, 4^{\prime \prime}\right.$-tetrahydroxydibenzofurandicarboxyl)]- $\beta$-D-glucopyranose.

Balanophotannin B (2), a tan powder, exhibited a caffeoyl group, a $1 \beta, 4,6-O$-tri-acylated glucopyranose moiety and two aromatic proton signals $(\delta 7.34,7.18)$ in its ${ }^{1} \mathrm{H}-\mathrm{NMR}$ spectrum. The ${ }^{1} \mathrm{H}$ - and ${ }^{13} \mathrm{C}$-NMR data (Table 1 ) of 2 closely resembled to those of 1-O- $(E)$-caffeoyl-4,6-(S)-HHDP- $\beta$-D-glucopyranose, a major ellagitannin existing in the aboveground part of Balanophora japonica, ${ }^{1)}$ especially the signals derived from the caffeoyl and glucose moieties are almost superimposable to those of 1-O- $(E)$-caffeoyl-4,6-(S)-HHDP- $\beta$ D-glucopyranose. Considering compound 2's molecular formula $\mathrm{C}_{29} \mathrm{H}_{22} \mathrm{O}_{16}$ obtained from the results of negative FABMS $\left(\mathrm{m} / \mathrm{z}: 625[\mathrm{M}-\mathrm{H}]^{-}\right)$and elemental analysis, 2 was deduced to be a dehydrated product of 1-O-(E)-caffeoyl-4,6$(S)$-HHDP- $\beta$-D-glucopyranose. Similarly, the appearance of 12 aromatic and 2 carbonyl carbon signals (Table 1 ) in the ${ }^{13} \mathrm{C}-\mathrm{NMR}$ spectrum of $\mathbf{2}$ indicated the presence of $1,1^{\prime}$ $\left(3,3^{\prime}, 4,4^{\prime}\right.$-tetrahydroxy) dibenzofurandicarboxyl group in the molecule. Thus, the structure of 2 was concluded to be 1-O(E)-caffeoyl-4,6-[1', $1^{\prime \prime}-\left(3^{\prime}, 3^{\prime \prime}, 4^{\prime}, 4^{\prime \prime}\right.$-tetrahydroxydibenzofurandicarboxyl)]- $\beta$-D-glucopyranose.
Table 1. ${ }^{13} \mathrm{C}-\mathrm{NMR}\left(75 \mathrm{MHz}\right.$, Acetone- $d_{6}$ ) Spectral Data of Compounds $\mathbf{1}$, $\mathbf{2}$ and $\mathbf{3}$

\begin{tabular}{rlll}
\hline \hline & $\mathbf{1}$ & $\mathbf{2}$ & $\mathbf{3}$ \\
\hline Caffeoyl-1 & - & 126.8 & 126.9 \\
2 & - & 114.9 & 115.0 \\
3 & - & 146.3 & 147.9 \\
4 & - & 149.3 & 149.4 \\
5 & - & 116.2 & 116.2 \\
6 & - & 122.9 & 123.0 \\
7 & - & 147.8 & 147.9 \\
8 & - & 113.9 & 113.9 \\
9 & - & 168.6 & 168.6 \\
Glc-1 & 96.6 & 94.8 & 96.5 \\
2 & 71.9 & 73.4 & 71.6 \\
3 & 74.7 & 73.8 & 74.2 \\
4 & 70.9 & 70.8 & 70.9 \\
5 & 74.2 & 76.2 & 74.8 \\
6 & 66.7 & 66.9 & 66.7 \\
$4,6-$ Acyl-1', $1^{\prime \prime}$ & $117.4,116.0$ & $116.1,115.2$ & $115.9,115.4$ \\
$2^{\prime}, 2^{\prime \prime}$ & $119.4,118.2$ & $119.7,116.8$ & $118.9,116.7$ \\
$3^{\prime}, 3^{\prime \prime}$ & $115.0,111.8$ & $114.7,111.6$ & $114.6,111.8$ \\
$4^{\prime}, 4^{\prime \prime}$ & $144.6,144.4$ & $144.8,144.4$ & $144.7,144.6$ \\
$5^{\prime}, 5^{\prime \prime}$ & $134.9,133.4$ & $135.0,133.0$ & $135.4,133.5$ \\
$6^{\prime}, 6^{\prime \prime}$ & $146.8,146.8$ & $146.8,146.9$ & $146.9,146.3$ \\
$7^{\prime}, 7^{\prime \prime}$ & $169.4,168.3^{a)}$ & $170.1,166.3$ & $169.6,166.1$ \\
Galloyl-1 $^{\prime \prime}$ & $121.3,120.4$ & - & 120.9 \\
2,6 & $110.4,110.2$ & - & 110.1 \\
3,5 & $146.0,146.0$ & - & 146.0 \\
4 & $139.5,139.0$ & - & 139.2 \\
7 & $166.2^{a}, 165.1$ & - & 166.7 \\
& & &
\end{tabular}

a) Assignments may be interchanged.

Balanophotannin C (3) which is also a tan powder purified from the same fraction together with compound $\mathbf{2}$, showed similarities with $\mathbf{2}$ in it ${ }^{1} \mathrm{H}$ - and ${ }^{13} \mathrm{C}-\mathrm{NMR}$ spectra. However, a galloyl's signal was observed in both ${ }^{1} \mathrm{H}$ - and ${ }^{13} \mathrm{C}$-NMR spectra of 3 , suggesting it has one more galloyl in the molecule than 2. This was supported by the negative FAB-MS of $\mathbf{3}$ which displayed an $[\mathrm{M}-\mathrm{H}]^{-}$molecular ion peak at $m / z: 777$. To elucidate the location of the galloyl group in the molecule of $\mathbf{3}$, the proton signals of the glucose moiety in $\mathbf{3}$ were fully assigned by using ${ }^{1} \mathrm{H}-{ }^{1} \mathrm{H}$ decoupling techniques. A comparison of the chemical shift of $\mathbf{3}$ and $\mathbf{2}$ led to a conclusion that 
the galloyl group is located in C-3 hydroxyl group of the glucose because a downshift $(\Delta \delta=+1.62 \mathrm{ppm})$ for H-3 was observed. Finally, transformation of compound 3 to 2 by tannase hydrolysis further confirmed its structure to be 1-O- $(E)$ caffeoyl-3-O-galloyl-4,6-[1', $1^{\prime \prime}-\left(3^{\prime}, 3^{\prime \prime}, 4^{\prime}, 4^{\prime \prime}\right.$-tetrahydroxydibenzofurandicarboxyl)]- $\beta$-D-glucopyranose. Its biogentic precursor $1-O-(E)$-caffeoyl-3- $O$-galloyl-4,6- $(S)$-HHDP- $\beta$-Dglucopyranose was also isolated from the same plant. ${ }^{1)}$

In summary, three ellagitannins $(\mathbf{1}-\mathbf{3})$ and four known lignan glcosides (4-7) were isolated from the extracts of fresh aboveground and underground parts of Balanophora japonica (Balanophoraceae) by column chromatographies. Balanophotannins $\mathrm{A}-\mathrm{C}(\mathbf{1}-\mathbf{3})$ have a $1,1^{\prime}-\left(3,3^{\prime}, 4,4^{\prime}\right.$ tetrahydroxy) dibenzofurandicarboxyl group linked to the 4,6-position of the glucose moiety. To the best of our knowledge, balanophotannins represent the first example of a type of ${ }^{4} \mathrm{C}_{1}$ conformation glucopyranose-containing ellagitannin having such acyl group in the molecule. Balanophotannins $\mathrm{A}-\mathrm{C}(\mathbf{1}-\mathbf{3})$ are the dehydrated products of the corresponding ellagitannins co-existing in the same plants. ${ }^{1)}$ In addition, the known lignan glycosides showed chemotaxonomic significance. Compound $\mathbf{5}$ was isolated from another parasitic medicinal plant Cynomorium songaricum (Cynomoriaceae) as a new lignan glycoside by the authors. ${ }^{2}$ A lignan glycoside with the same dihydrobenzofuran skeleton as balanophonin 4- $O$ - $\beta$-D-glucopyranoside (4) was also found in Cynomorium songaricum. ${ }^{2)}$ Recently, balanophonin $4-O-\beta$-Dglucopyranoside (4) and related lignan glycosides were isolated from a parasitic plant Aeginetia indica (Orobanchaceae). These chemical information may be regarded as the evidence for supporting the taxonomic affinities among families Balanophoraceae, Cynomoriaceae and Orobanchaceae.

\section{Experimental}

General Optical rotations were measured with a JASCO DIP-370 digital polarimeter. ${ }^{1} \mathrm{H}$ - and ${ }^{13} \mathrm{C}-\mathrm{NMR}$ spectra were recorded on Varian Unity plus 500 and Varian Gemini 300 spectrometers. Coupling constants $(J)$ are expressed in $\mathrm{Hz}$, and chemical shifts are given on a $\delta(\mathrm{ppm})$ scale with tetramethylsilane as an internal standard. MS were recorded on a JEOL JMS DX-303 spectrometer, and glycerol was used as a matrix for FAB-MS measurement. Column chromatographies were performed with Kieselgel 60 (70-230 mesh, Merck), Sephadex LH-20 (25-100 $\mu \mathrm{m}$, Pharmacia Fine Chemical Co. Ltd.), MCI-gel CHP 20P (75-150 $\mu \mathrm{m}$, Mitsubishi Chemical Co. Ltd.), TSK gel Toyopearl HW-40F (Tosoh), Chromatorex ODS (100200 mesh, Fuji Silysia Chemical), Bondapak C18 (125 $\mu \mathrm{m}$, Waters). Thin layer chromatography (TLC) was performed on precoated Kieselgel $60 \mathrm{~F}_{254}$ plates $(0.2 \mathrm{~mm}$ thick, Merck), and spots were detected by ultraviolet (UV) illumination and by spraying $10 \%$ sulfuric acid reagent.

Extraction and Isolation (a) Aboveground Part: The $\mathrm{MeOH}$ extract $(223.7 \mathrm{~g})$ of the aboveground part of fresh Balanophora japonica $(1.73 \mathrm{~kg})$ collected in Nagasaki Prefecture was divided into $\mathrm{Et}_{2} \mathrm{O}$, EtOAc and $\mathrm{H}_{2} \mathrm{O}$ layers as described in our previous paper. ${ }^{1)}$ The EtOAc layer was subjected to Toyopearl HW-40 column chromatography $\left(20-100 \% \mathrm{MeOH}\right.$ in $\left.\mathrm{H}_{2} \mathrm{O}\right)$ to give three fractions. The third fraction which was eluated by $80-100 \%$ $\mathrm{MeOH}$ was sequentially chromatographed over Chromatorex ODS $\left(\mathrm{H}_{2} \mathrm{O}-\mathrm{MeOH}\right)$, Sephadex LH-20 $(80-100 \% \mathrm{MeOH})$ and MCI-gel CHP $20 \mathrm{P}$ $\left(\mathrm{H}_{2} \mathrm{O}-\mathrm{MeOH}\right)$ to furnish compounds $1(16.2 \mathrm{mg}), 2(88.4 \mathrm{mg})$ and 3 (14.5 mg).

(b) Underground Part: The $\mathrm{MeOH}$ extracts $(131 \mathrm{~g})$ of fresh underground part $(1.01 \mathrm{~kg})$ of $B$. japonica was separated into four fractions by silica gel chromatography as described in our previous paper. ${ }^{1)}$ Compound 6 (2.12 g) was obtained from the second fraction $(11.6 \mathrm{~g})$ by recrystallization. The third fraction $(41.0 \mathrm{~g})$ was repeatedly chromatographed over MCI-gel CHP 20P, Sephadex LH-20 and TSK gel Toyopearl HW-40F (all eluted with $\mathrm{H}_{2} \mathrm{O}$ containing an increasing amount of $\mathrm{MeOH})$ to afford compounds $\mathbf{4}(15.2 \mathrm{mg}), \mathbf{5}$ $(421.8 \mathrm{mg})$ and 7 (197.2 $\mathrm{mg})$.

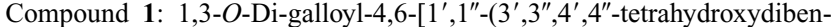
zofurandicarboxyl)]- $\beta$-D-glucopyranose: $\mathrm{A} \tan$ amorphous powder, $[\alpha]_{\mathrm{D}}^{20}$ $+12.2^{\circ}(c=0.4, \mathrm{MeOH})$. Anal. Calcd for $\mathrm{C}_{34} \mathrm{H}_{24} \mathrm{O}_{21} \cdot 9 / 2 \mathrm{H}_{2} \mathrm{O}: \mathrm{C}, 48.07 ; \mathrm{H}$, 3.92. Found: C, 48.08; H, 4.06. Negative FAB-MS $m / z$ : $767[\mathrm{M}-\mathrm{H}]^{-} .{ }^{1} \mathrm{H}-$ NMR (300 MHz, $\left.\mathrm{CD}_{3} \mathrm{OD}\right): \delta 7.31,6.74$ (each $1 \mathrm{H}$, s, tetrahydroxydibenzofurandicarboxyl-3', $\left.3^{\prime \prime}\right), 7.25,7.21$ (each $2 \mathrm{H}$, s, galloyls), $6.02(1 \mathrm{H}, \mathrm{d}, J=8 \mathrm{~Hz}$, glc-1), $5.68(1 \mathrm{H}, \mathrm{t}, J=9 \mathrm{~Hz}$, glc- 3$), 5.34(1 \mathrm{H}, \mathrm{t}, J=9 \mathrm{~Hz}$, glc- -4$), 4.90(1 \mathrm{H}$, dd, $J=9,11 \mathrm{~Hz}$, glc-6a), $4.26(1 \mathrm{H}, \mathrm{m}$, glc-5), $4.14(1 \mathrm{H}, \mathrm{dd}, J=8,9 \mathrm{~Hz}$, glc2), $4.08(1 \mathrm{H}, \mathrm{m}$, glc- $6 \mathrm{~b}) .{ }^{13} \mathrm{C}$-NMR data see Table 1.

Tannase Hydrolysis of 1 A solution of $1(5 \mathrm{mg})$ in water $(1 \mathrm{ml})$ was shaken with tannase (kindly provided by Dr. M. Kanaoka, Sankyo Co. Ltd.) at $37{ }^{\circ} \mathrm{C}$ for $30 \mathrm{~min}$. The mixture was applied to $\mathrm{MCI}$-gel CHP20P column chromatography with water containing increasing proportions of $\mathrm{MeOH}$ to yield compound 1a $(3 \mathrm{mg})$ : 4,6-[1', $1^{\prime \prime}-\left(3^{\prime}, 3^{\prime \prime}, 4^{\prime}, 4^{\prime \prime}\right.$-tetrahydroxydibenzofurandicarboxyl)]-glucose. A tan amorphous powder, $[\alpha]_{\mathrm{D}}^{24}+31.2^{\circ}(c=0.2$, $\mathrm{MeOH}$ ). Anal. Calcd for $\mathrm{C}_{20} \mathrm{H}_{16} \mathrm{O}_{13} \cdot 4 \mathrm{H}_{2} \mathrm{O}: \mathrm{C}, 44.78 ; \mathrm{H}, 4.51$. Found: $\mathrm{C}$, 44.72; H, 4.54. Negative FAB-MS $m / z: 463[\mathrm{M}-\mathrm{H}]^{-}$. ${ }^{1} \mathrm{H}-\mathrm{NMR}(300 \mathrm{MHz}$, acetone- $\left.d_{6}\right): \delta 7.30(2 \mathrm{H}), 7.14,7.13\left(\right.$ total $\left.4 \mathrm{H}, \mathrm{s}, \mathrm{H}-3^{\prime}, \mathrm{H}-3^{\prime \prime}\right), 5.20(1 \mathrm{H}, \mathrm{d}$, $J=4 \mathrm{~Hz}, \alpha$-glc-1), $4.90(2 \mathrm{H}, \mathrm{t}, J=9 \mathrm{~Hz}, \alpha$-glc- $4, \beta$-glc- 4$), 4.71(2 \mathrm{H}, \mathrm{t}$, $J=11 \mathrm{~Hz}, \alpha$-glc-6a, $\beta$-glc-6a), $4.65(1 \mathrm{H}, \mathrm{d}, J=8 \mathrm{~Hz}, \alpha$-glc-1), $4.20(1 \mathrm{H}, \mathrm{m}$, $\alpha$-glc-5), 4.04 (1H, t, $J=9 \mathrm{~Hz}, \alpha$-glc-3), 3.97 (1H, m, $\beta$-glc-5), 3.90, 3.80 (each $1 \mathrm{H}, \mathrm{dd}, J=8,11 \mathrm{~Hz}, \alpha$-glc-6b, $\beta$-glc-6b), $3.79(1 \mathrm{H}, \mathrm{t}, J=9 \mathrm{~Hz}, \beta$-glc3), $3.57(1 \mathrm{H}, \mathrm{dd}, J=4,9 \mathrm{~Hz}, \alpha$-glc-2), $3.31(1 \mathrm{H}, \mathrm{t}, J=9 \mathrm{~Hz}, \beta$-glc- 2$)$.

Compound 2: 1-O- $(E)$-Caffeoyl-4,6-[1', $1^{\prime \prime}-\left(3^{\prime}, 3^{\prime \prime}, 4^{\prime}, 4^{\prime \prime}\right.$-tetrahydroxydibenzofurandicarboxyl) $]$ - $\beta$-D-glucopyranose: A tan amorphous powder, $[\alpha]_{\mathrm{D}}^{16}$ $+82.3^{\circ}(c=0.2, \mathrm{MeOH})$. Anal. Calcd for $\mathrm{C}_{29} \mathrm{H}_{22} \mathrm{O}_{16} \cdot 7 / 4 \mathrm{H}_{2} \mathrm{O}: \mathrm{C}, 52.93 ; \mathrm{H}$, 3.91. Found: C, 52.74; H, 4.00. Negative FAB-MS m/z: $625[\mathrm{M}-\mathrm{H}]^{-} .{ }^{1} \mathrm{H}-$ NMR $\left(300 \mathrm{MHz}\right.$, acetone- $\left.d_{6}\right): \delta 7.34,7.18$ (each $1 \mathrm{H}$, s, tetrahydroxydibenzofurandicarboxyl-3',3"), $7.23(1 \mathrm{H}, \mathrm{d}, J=2 \mathrm{~Hz}$, caf-2), $6.90(1 \mathrm{H}, \mathrm{d}, J=8 \mathrm{~Hz}$, caf-5), $7.07(1 \mathrm{H}, \mathrm{dd}, J=2,8 \mathrm{~Hz}$, caf-6), $7.68(1 \mathrm{H}, \mathrm{d}, J=16 \mathrm{~Hz}$, caf-7), 6.36 $(1 \mathrm{H}, \mathrm{d}, J=16 \mathrm{~Hz}$, caf- 8$), 5.76(1 \mathrm{H}, \mathrm{d}, J=8 \mathrm{~Hz}, \mathrm{glc}-1), 3.73(1 \mathrm{H}, \mathrm{dd}, J=8$, $9 \mathrm{~Hz}$, glc-2), $4.03(1 \mathrm{H}, \mathrm{t}, J=9 \mathrm{~Hz}$, glc-3), $5.04(1 \mathrm{H}, \mathrm{t}, J=9 \mathrm{~Hz}$, glc- 4$), 4.05$ $\left(1 \mathrm{H}, \mathrm{m}\right.$, glc-5), $4.78\left(1 \mathrm{H}, \mathrm{dd}, J=9,11 \mathrm{~Hz}\right.$, glc-6a), $4.05(1 \mathrm{H}, \mathrm{m}, \mathrm{glc}-6 \mathrm{~b}) .{ }^{13} \mathrm{C}-$ NMR data see Table 1.

Compound 3: 1-O-(E)-Caffeoyl-3-O-galloyl-4,6-[1', $1^{\prime \prime}-\left(3^{\prime}, 3^{\prime \prime}, 4^{\prime}, 4^{\prime \prime}\right.$-tetrahydroxydibenzofurandicarboxyl)]- $\beta$-D-glucopyranose: A tan amorphous powder, $[\alpha]_{\mathrm{D}}^{16}+81.7^{\circ}(c=0.5, \mathrm{MeOH})$. Anal. Calcd for $\mathrm{C}_{36} \mathrm{H}_{26} \mathrm{O}_{20} \cdot 2 \mathrm{H}_{2} \mathrm{O}: \mathrm{C}$, 53.08; H, 3.71. Found: C, 53.24; H, 3.90. Negative FAB-MS m/z: 777 $[\mathrm{M}-\mathrm{H}]^{-}$. ${ }^{1} \mathrm{H}-\mathrm{NMR}\left(500 \mathrm{MHz}, \mathrm{CD}_{3} \mathrm{OD}\right): \delta 7.31,6.74$ (each $1 \mathrm{H}$, s, tetrahydroxydibenzofurandicarboxyl-3', $\left.3^{\prime \prime}\right), 7.26(2 \mathrm{H}, \mathrm{s}$, galloyl $), 7.23(1 \mathrm{H}, \mathrm{d}$, $J=2 \mathrm{~Hz}$, caf-2), $6.90(1 \mathrm{H}, \mathrm{d}, J=8 \mathrm{~Hz}$, caf-5), $7.07(1 \mathrm{H}, \mathrm{dd}, J=2,8 \mathrm{~Hz}$, caf-6), $7.72(1 \mathrm{H}, \mathrm{d}, J=16 \mathrm{~Hz}$, caf-7), $6.37(1 \mathrm{H}, \mathrm{d}, J=16 \mathrm{~Hz}$, caf -8$), 5.95(1 \mathrm{H}, \mathrm{d}$, $J=8 \mathrm{~Hz}$, glc- 1$), 4.05(1 \mathrm{H}, \mathrm{dd}, J=8,9 \mathrm{~Hz}$, glc-2), $5.65(1 \mathrm{H}, \mathrm{t}, J=9 \mathrm{~Hz}$, glc- 3 ), $5.33(1 \mathrm{H}, \mathrm{t}, J=9 \mathrm{~Hz}$, glc-4), $4.25(1 \mathrm{H}, \mathrm{m}$, glc -5$), 4.89(1 \mathrm{H}, \mathrm{dd}, J=9,11 \mathrm{~Hz}$, glc-6a), $4.08\left(1 \mathrm{H}, \mathrm{m}\right.$, glc-6b). ${ }^{13} \mathrm{C}-\mathrm{NMR}$ data see Table 1.

Tannase Hydrolysis of 3 A solution of $\mathbf{3}(10 \mathrm{mg})$ in water $(2 \mathrm{ml})$ was hydrolyzed in a manner similar to that described for $\mathbf{1}$ to yield compound $\mathbf{2}$ $(5.9 \mathrm{mg})$.

Acknowledgments The authors are grateful to Mr. K. Inada, Mr. N. Yamaguchi and Mr. M. Ohwatari of the Center for Instrumental Analysis of Nagasaki University for NMR, MS measurements and elemental analyses, respectively. This research was partially supported by the Faculty Research Grants from Hong Kong Baptist University.

\section{References}

1) Jiang Z.-H., Hirose Y., Iwata H., Sakamoto S., Tanaka T., Kouno I., Chem. Pharm. Bull., 49, 887-892 (2001).

2) Jiang Z.-H., Tanaka T., Sakamoto M., Jiang T., Kouno I., Chem. Pharm. Bull., 49, 1036-1038 (2001).

3) Ho J.-C., Chen C.-M., Row L.-C., J. Chinese Chemical Society (Taipei, Taiwan), 50, 1271-1274 (2003).

4) Yuen M. S. M., Xue F., Mak T. C. W., Wong H. N. C., Tetrahedron, 54, 12429-12444 (1998).

5) Haruna M., Koube T., Ito K., Murata H., Chem. Pharm Bull., 30, 1525-1527 (1982).

6) Casabuono A. C., Pomilio A. B., Phytochemistry, 34, 479-483 (1994).

7) Qiu S.-X., Lu Z.-Z., Luyengi L., Lee S.-K., Pezzuto J. M., Farnsworth N. R., Thompson L. U., Fong H. H. S., Pharm. Biol., 37, 1-7 (1999).

8) Saijo R., Nonaka G., Nishioka I., Chem. Pharm. Bull., 37, $2063-$ 2070 (1989).

9) Zhang Y.-J., Abe T., Tanaka T., Yang C.-R., Kouno I., J. Nat. Prod., 64, $1527-1532$ (2001). 